\title{
Scanpath analysis of expertise and culture in teacher gaze in real-world classrooms
}

\author{
Nora A. McIntyre ${ }^{1}$ (D) Tom Foulsham ${ }^{2}$
}

Received: 23 December 2016/ Accepted: 26 December 2017/Published online: 5 January 2018

(C) The Author(s) 2018. This article is an open access publication

\begin{abstract}
Humans are born to learn by understanding where adults look. This is likely to extend into the classroom, making teacher gaze an important topic for study. Expert teacher gaze has mainly been investigated in the laboratory, and has focused mostly on one cognitive process: teacher attentional (i.e., information-seeking) gaze. No known research has made direct cultural comparisons of teacher gaze or successfully found expert-novice differences outside Western settings. Accordingly, we conducted a real-world study of expert teacher gaze across two cultural settings, exploring communicative (i.e., information-giving) as well as attentional gaze. Forty secondary school teachers wore eye-tracking glasses, with 20 teachers (10 expert; 10 novice) from the UK and 20 teachers (10 expert; 10 novice) from Hong Kong. We used a novel eye-tracking scanpath analysis to ascertain the importance of expertise and culture, individually and as a combination. Attentional teacher scanpaths were significantly more similar within than across expertise and expertise + culture sub-groups; communicative scanpaths were significantly more similar within than across expertise and culture. Detailed analysis suggests that (1) expert teachers refer back to students constantly through focused gaze during both attentional and communicative gaze and that (2) expert teachers in Hong Kong scan students more than experts do in the UK.
\end{abstract}

Keywords Real-world - Teacher expertise - Cross-cultural comparisons · Eye-tracking · Scanpath analysis

Nora A. McIntyre

n.mcintyre@sheffield.ac.uk

1 Department of Psychology, University of Sheffield, Sheffield S1 2LT, UK

2 Department of Psychology, University of Essex, Colchester CO4 3SQ, UK 


\section{Introduction}

Expertise can be seen at every level of teaching. At the macro-level, expert teachers demonstrate stronger knowledge, organisation and reflectiveness (e.g., Allen and Casbergue 1997). At the micro-level, teachers reveal exceptional and unique internal processes that have been developed and refined over time, including their memory (Ericsson and Kintsch 1995), strategy (Chassy and Gobet 2011), efficiency (Haider et al. 2005) and intuition (Sherin 2006). Likewise, expertise in teaching not only predicts what teachers look at but also suggests how teachers look around the classroom (e.g., Cortina et al. 2015).

It has generally been proposed that gaze sequences-or 'scanpaths'-reflect internal representations of the world, namely 'cognitive models' (Henderson 2003; Choi et al. 1995). We can therefore expect expertise to differentiate teachers' scanpaths as it does in other domains (e.g., Foerster et al. 2011). Since culture entails different experiences, culture might also differentiate teachers' scanpaths, which would support broader theories of expertise (Sternberg 2014). We therefore investigated the role of expertise and culture in distinguishing teachers' scanpaths.

So far, most expertise and teacher gaze research has focused on information-seeking, or attentional, gaze (Reingold and Sheridan 2011). Such research is relevant to the passive processes of observation, when viewers are looking for particular details or waiting to receive knowledge so that information is going from the scene (or audience) to the viewer. Yet, information-giving, or communicative, gaze is important to consider in classroom research, given the role of gaze in human learning (Csibra 2010), social interaction (Wu et al. 2014), and successful teaching (Leinhardt 1987). Unlike in attentional gaze, the viewer is active in transmitting knowledge to an audience such that information is now traveling from the viewer to the scene (or audience). It has been proposed that complex social processes are best explored in the real-world (Fiske and Taylor 2013). Although some research has examined the gaze of expert teachers (e.g., Cortina et al. 2015), this behaviour may be best investigated in the real-world due to the complex nature of teaching (Berliner 2001) and the domain-specificity of expertise in general (Ericsson 2014). Together, the present study addressed gaps in previous investigations by examining the role of expertise and culture in teachers' scanpaths during both attentional and communicative gaze, in the real-world, across two cultural settings.

While there are very few datasets regarding gaze in real-world teaching, we recently reported a study in which teachers from the UK and Hong Kong were eye-tracked in secondary school classrooms. We used aggregate measures of dynamic properties in teacher gaze to compare the variability of expert teachers' gaze in comparison with novices' (McIntyre et al. 2017). This analysis provided some of the first information about teachers' real-world gaze, but was limited by its reliance on aggregate measures of where teachers look: such measures are unsuitable for examination of the "cognitive model' underlying teacher gaze. Here, we provide a new analysis of data from the same study by testing dynamic measures of teacher gaze, that is the gaze sequences of the same teachers which, it has been argued, are critical reflections of the cognitive model. Our expectation was that this new approach would reveal additional details about teacher expertise. 


\section{Scanpath analysis}

Despite recent interest in teacher gaze, there has been little investigation of the underlying structure of teachers' gaze patterns. A scanpath is a "repetitive sequence of saccades and fixations, idiosyncratic to a particular subject [person] and to a particular target pattern" (Choi et al. 1995, p. 450). Scanpath analysis is therefore the investigation of gaze sequences, which preserves information about what is being looked at and the order in which these looks occur. It has been argued that social interactions (Bakeman and Gottman 1997; Hewes 1979; Sackett 1987) —and therefore teaching (Palincsar 1998; Pianta et al. 2012; Wubbels et al. 2016) — are inherently sequential and dynamic, since each social act needs to be understood in the context of earlier behaviours. Thus, teacher scanpaths are likely to contain rich sequential patterns which have yet to be investigated.

Scanpath analysis is a particularly valuable approach in teaching research, because a sequence of gaze acts is normally involved during pedagogical exchanges. To begin with, a learner (e.g., an infant) is often invited into an educational episode when the teacher (e.g., the adult carer) catches his or her attention through a social signal which usually involves eye contact (Batki et al. 2000; Committeri et al. 2015; Farroni et al. 2002). A process of shared attention (Baron-Cohen 1995) is thus initiated, in which the teacher's gaze shift redirects learner attention to the right gaze target (de Langavant et al. 2011; Senju and Csibra 2008) and triggers 'gaze following' in the learner as they are drawn to shift their gaze to where the teacher has shifted their attention (Böckler et al. 2015; Farroni et al. 2004). When both teacher and learner are looking at the same thing, shared attention is achieved and the intended teaching can be given regarding the shared gaze target (Csibra and Gergely 2009; Tomasello 2000). A sequence of multiple gaze targets is therefore involved in each pedagogical episode. Conventional aggregated analyses, which look only at the overall amount of time looking at different targets, may miss such patterns.

One of the most common techniques for scanpath analysis involves the representation of gaze sequences as letter strings. The similarity between two strings is represented by the 'string edit distance' (SED; Brandt and Stark 1997; cf. Levenshtein 1966). A SED is calculated by counting the number of edits-insertions, deletions and substitutions-before two strings become identical. This procedure is flexible, meaning that strings can be defined by geometric gaze coordinates, areas of interest, or semantic codes. The present study used this last approach, since the data was from real-world, mobile eye-tracking which was then coded in order to identify each gaze target.

\section{Culture-specific expertise in teacher scanpaths}

Expertise is shown through consistent traits across professions (Sternberg and Horvath 1995) while each individual's area of expertise is domain-specific (Bédard and Chi 1992). In teaching, experts are more knowledge-driven, efficient, flexible during lessons and more consistent across lessons (McIntyre et al. 2017). Culture, however, plays a central role in defining teacher expertise. As with any profession, 'expertise' in teaching is embedded in contextual policies (Berliner 2001) and culture (Sternberg 2014). Compared with East Asia, learning in the West involves more vocal input from the student (even disagreement, Hofstede 1986), more group work (Leung 1995) and a more analytic approach to learning (Yang and Cobb 1995). Student preferences diverge across cultures (Wozniaková 2016), as do their expectations of their teachers (Zhang et al. 2005). Teachers' strengths also differ across cultures, with Western teachers excelling in general pedagogical knowledge and 
East Asian teachers excelling in subject and pedagogical content knowledge (König et al. 2011).

It has been proposed that scanpaths reflect "the read-out of the internal representation of pictures, the so-called 'cognitive model'" (Choi et al. 1995). This scanpath theory proposes that some internal process drives each person to look where they do, in the order that they do. Because each individual has their own unique cognitive model, scanpaths of the same individual on multiple occasions will be more similar when compared than scanpaths of different individuals. If scanpaths are indeed more guided by cognitive models, experts should produce significantly different scanpaths to novices. With expertise, observers gain in experience- and knowledge-informed cognitive models and teachers' cognitive models are likely to become more refined than novices'. The scanpaths of expert teachers should therefore be more knowledge-driven and effective than those of novices, providing a template of what to think about, and in what order, for novices' successful professional development.

Given that scanpaths are affected by experience, culture can also be expected to shape scanpaths. Through the course of professional development, teachers are likely to treat different classroom regions with differing task-relevance and appropriateness according to their own culture (e.g., Hofstede 1986), such that the most important regions changes with culture (e.g., Berliner 2001). Teacher scanpaths should thus reflect culture-specific cognitive models, especially expert teachers'. Teachers from different cultures should therefore produce significantly different scanpaths from each other, while individuals should display similar scanpaths to teachers belonging to the same cultural group. Using expert scanpaths from their own culture, novices can more accurately emulate an order of what to consider in classroom teaching that is better rooted in the values in their own cultural setting.

\section{The present article}

The present article aims to apply scanpath methods and comparison to teacher gaze, by expanding scanpath research into differences across expertise levels (e.g., Humphrey and Underwood 2009) to the teaching profession and by extending scanpath comparisons across cultures. The present analysis also extends our previous work on the same dataset (e.g., McIntyre et al. 2017) in which we reported that expert teachers give greater importance to students and use their classroom gaze more efficiently (i.e., primarily using the most relevant gaze) than novices. Experts were also more strategically consistent. Cultural differences were found, with UK teachers showing more attentional efficiency and Hong Kong teachers more communicative efficiency. Although this previous analysis used dynamic measures, their differences still involved aggregating over many didactic events. We anticipated that the present study, which instead adopts a sequential and fully dynamic perspective, would shed further light on expertise and culture in teacher gaze. This will allow us to understand how expertise and culture affect moment-to-moment changes in behaviour (i.e., teacher scanpaths) and the proposed underlying cognitive model. We had a number of specific hypotheses based on the expectation that expertise and culture will affect teachers' looking behaviour.

Hypothesis 1 We expected scanpaths to be significantly more similar when compared within an individual than when compared across individuals. This finding would give credence to subsequent comparisons, and it would support the idea that teachers show an idiosyncratic strategy. 
Hypothesis 2 Since teachers with the same expertise level are more likely to share a cognitive model than teachers of differing expertise, scanpaths of expert teachers should be more similar when compared with other experts than when compared with novice teachers.

Hypothesis 3 Since the cognitive model of teachers is likely to be similar within a cultural group, teacher scanpaths should be more similar when compared within cultures than when scanpaths are compared across cultural settings.

Hypothesis 4 Cognitive models are likely to be most similar when teachers have the same expertise level and cultural setting. Therefore, scanpaths within the same expertise and cultural group were expected to be significantly more similar than those of teachers in different expertise and cultural group.

\section{Method}

\section{Participants}

Participants consisted of 20 Hong Kong Chinese (henceforth East Asian) and 20 White Caucasian UK (henceforth Western European) secondary school teachers. Schools were selected on the condition that they followed their respective national curricula and that they consisted of students from the first to fifth years of secondary education: two schools were sampled in each country.

Cultural groupings in the present study were based on geographical location (i.e., in Hong Kong vs. in the UK). Each cultural group were ethnically homogenous, with the Hong Kong sample comprising entirely of Hong Kong Chinese and the UK sample entirely of White British teachers and students. Expert teachers were defined using the guidelines given by Palmer et al. (2005), which consisted of (a) having at least 6 years' experience, (b) social nomination as an expert in teaching (selected by the school leadership), (c) professional memberships, and (d) performance ratings (based on in-school classroom observations). According to MANOVA, teachers who were nominated as experts (criterion b) significantly differed on years' experience (criterion a), professional memberships (criterion c) and performance ratings (criterion d), $F(3,37)=14.22, p<0.001, \eta_{p}^{2}=0.54$. See Table 1 for detailed teacher demographics. A previous report indicated that differences

Table 1 Teacher demographics

\begin{tabular}{|c|c|c|c|c|c|c|c|c|c|c|c|c|c|c|}
\hline \multirow[t]{2}{*}{ Culture } & \multirow[t]{2}{*}{ Expertise } & \multirow[t]{2}{*}{$N$} & \multicolumn{2}{|l|}{ Age } & \multicolumn{2}{|c|}{ Gender } & \multicolumn{4}{|c|}{ Years' experience } & \multicolumn{2}{|c|}{$\begin{array}{l}\text { Perf } \\
\text { Ratings }\end{array}$} & \multicolumn{2}{|c|}{ Add Quals } \\
\hline & & & $M$ & $S D$ & M & $\mathrm{F}$ & $M$ & $S D$ & Min & Max & $M$ & $S D$ & $M$ & $S D$ \\
\hline \multirow[t]{2}{*}{ HK } & Expert & 10 & 44.00 & 9.94 & 3 & 7 & 19.30 & 7.47 & 10 & 32 & 1.60 & 0.84 & 2.70 & 1.49 \\
\hline & Novice & 10 & 26.00 & 3.16 & 3 & 7 & 4.60 & 3.24 & 1 & 10 & 2.70 & 0.95 & 1.10 & 1.10 \\
\hline \multirow[t]{2}{*}{ UK } & Expert & 10 & 35.00 & 8.16 & 4 & 6 & 11.00 & 7.36 & 3 & 28 & 1.20 & 0.42 & 2.10 & 0.74 \\
\hline & Novice & 10 & 33.00 & 10.33 & 4 & 6 & 3.23 & 2.48 & 2 & 10 & 2.09 & 0.70 & 1.27 & 0.65 \\
\hline
\end{tabular}

'Perf Ratings' = abbreviated performance ratings which are reverse-scored ( 1 bring 'Outstanding'; 4 being 'Inadequate'); 'Add Quals' = abbreviated additional qualifications 
between culture and expertise remain even when teacher age and subject are controlled (McIntyre et al. 2017).

\section{Apparatus}

The Tobii 1.0 glasses eye-tracker was used to record teacher gaze. This eye-tracker was monocular, with a sampling rate of $30 \mathrm{~Hz}$ (i.e., 30 frames per second) and calibrated using nine gaze points. The eye-tracker yielded a 640 by $480 \mathrm{px}$ video, capturing $56^{\circ}$ horizontally and $40^{\circ}$ vertically, as well as an audio recording. Three approaches were used to secure quality of data analysis: a parallax correction tool is provided with our eye-tracking package, Tobii Studio 3.2.1, to reduce risks associated with monocular eye-trackers; each participant was asked to confirm the location of the gaze cursor during cued retrospective reporting (van Gog et al. 2005); when the gaze cursor disappeared, we applied the code, Unsampled.

\section{Procedure}

The teachers wore eye-tracking glasses during one 10-min 'teacher-centred' segment of their own lesson. That is, when the teacher was at the centre of the whole class' attention: introducing an activity, explaining new concepts, or presenting new material. This lesson followed on from each teacher's original plans for curriculum delivery; we simply waited for a teacher-centred section in the lesson scheduled for data collection to install the eyetracker onto the participating teacher. As such, each participant's teacher-centred segment differed from each other. The eye-tracking glasses were calibrated by the researcher just before recordings took place. In order to preserve the individual calibration, participants were instructed not to move the glasses until recording was over. Once $10 \mathrm{~min}$ of teachercentred learning was recorded, the researcher waited for a considerate moment to remove the eye-tracking equipment from the teacher.

\section{Analysis and Results}

\section{Coding}

We systematically coded teacher gaze and simultaneous verbalisations. Both the teacher gaze and simultaneous verbalisations were coded from the start to the end of analysed periods of eye-tracking.

\section{Gaze codes}

Gaze behaviour was coded by the researcher by slowing the playback to one eighth of realtime speed and manually applying the gaze behaviour codes. The gaze behaviours coded were student fixations (i.e., focused gaze at students), student scan, student material, teacher material, other (i.e., miscellaneous) and unsampled gaze. The student fixation code was applied when the gaze cursor overlaid students for more than four frames. Student scans were student-oriented gaze, during which the gaze cursor overlaid students for less than four frames (Franchak et al. 2011; Hanley et al. 2015). Unsampled gaze was coded when the gaze cursor disappeared from gaze replay. 


\section{Didactic codes}

Existing theories conceptualise teacher activity too broadly for the present data (i.e., different activity types not teacher-centred activity only, Berliner 2004; Hofstede 1986; Leung 1995; Pianta et al. 2004), in too much detail (i.e., speech acts within each teacher utterance, Searle 1969) or in topics that are too specific for the present article (e.g., classroom management not information-seeking vs. information-giving in general, Elliott et al. 2011). A coding scheme was therefore developed in a bottom-up manner, based on participating teachers' verbalisations rather than an existing theory.

Simultaneous verbal data was coded manually while playing the video in real-time (i.e., full playback speed) to generate teacher didactic codes. The simultaneous verbal data from eye-tracking recordings was divided into five didactic behaviours: address behaviour (i.e., directly instructing students to change their behaviour), attention (i.e., student or teacher asking and answering questions), communication (i.e., teachers lecturing), refer notes (i.e., teacher referring to presentation slides or students' resources), logistics (e.g., teacher moving the presentation onto another slide). In particular, questioning only consisted of periods of dialogue: that is, question-and-answering between teacher and students, while the teacher was at the front. Questioning thus included classroom silence as the teacher waited for students to answer their question; it also included periods when students spoke instead of the teacher. Talking consisted of straight talk and rhetorical questioning by the teacher. We thus followed McNeill's (2006) approach by interpreting non-verbal behaviour using simultaneous verbalisations.

\section{Didactic events}

Because attentional and communicative gaze were dramatically more common among teachers, the present study focused on these two didactic events only. Since social interaction is integral to classroom teaching (e.g., Pianta et al. 2012), attentional and communicative gaze were regarded as the most relevant teacher gaze to be analysed (cf. McIntyre et al. 2017). Of all the didactic behaviours, the present research explored only two-attention and communication - and six gaze codes were possible for each didactic behaviour, resulting in a total of 12 didactic events.

\section{Scanpaths}

For each participant, we generated multiple scanpaths (i.e., gaze event sequences) starting from the first ten gaze behaviours occurring within each period of either attentional or communicative teaching. In opting for ten (as in Foulsham et al. 2012; rather than five, e.g., Freeth et al. 2011), we hoped to capture greater detail in teachers' gaze behaviour. In using the first ten behaviours, we aimed to achieve more comparability between teachers than if no limit was set on the start-point. Thus, for each participant, we generated attentional gaze scanpaths and communicative gaze scanpaths. To illustrate, one attentional scanpath of one participant is depicted in Fig. 1. 'Unsampled' gaze targets were excluded from scanpaths. During the 10-min recording time, each participant took part in multiple episodes of questioning and talking, so each participant's data yielded several scanpaths.

Sequences of gaze events were compared using the string edit distance (SED; Brandt and Stark 1997), which is also known as the Levenshtein (1966) distance. The algorithm 

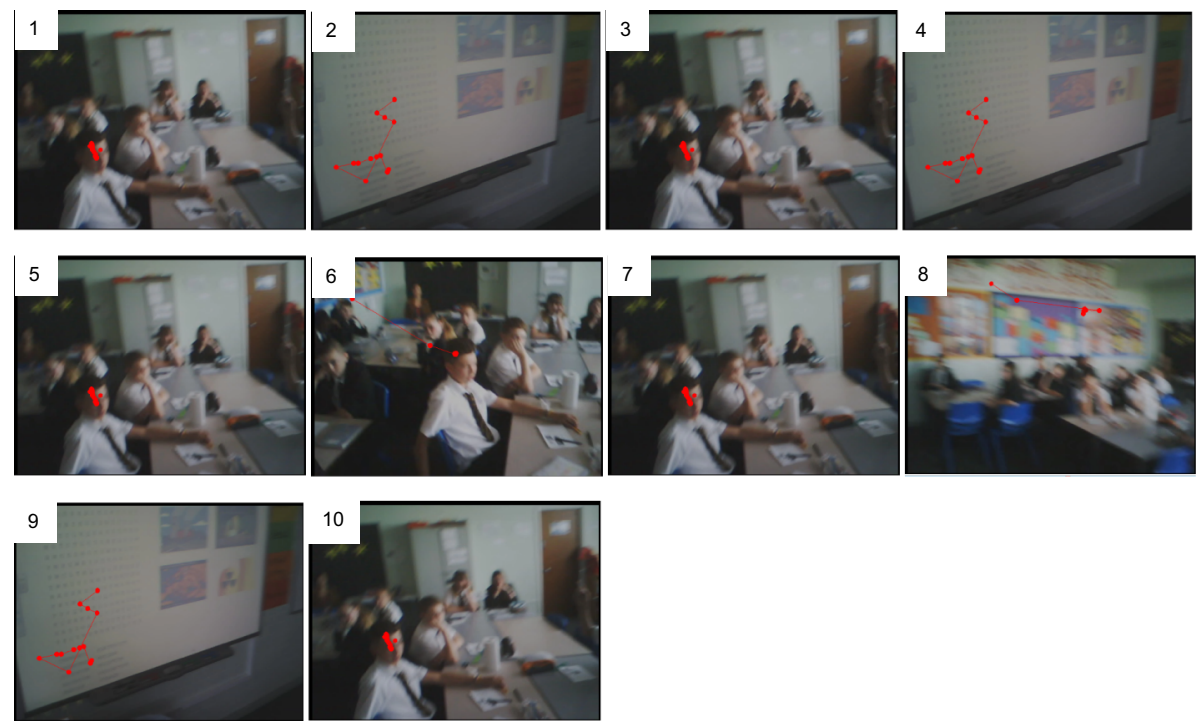

Fig. 1 Attentional scanpath of a UK expert (Participant 24) used for scanpath comparisons. This sequence of gaze targets show an attentional scanpath of $\mathbf{1}$ student fixation, $\mathbf{2}$ teacher material, $\mathbf{3}$ student fixation, $\mathbf{4}$ teacher material, $\mathbf{5}$ student fixation, $\mathbf{6}$ student scan, $\mathbf{7}$ student fixation, $\mathbf{8}$ other, $\mathbf{9}$ teacher material then, finally, 10 student fixation. The sequence of gaze events can be represented with the scanpath string, FTFTFCFOTF

for calculating the distance between two strings is widely available, and yields the minimum number of changes required to turn one string into the other. This number was divided by the length of the longer string and then subtracted from 1 to give a similarity score ranging from 0 to 1,1 being 'most similar' (i.e., identical).

\section{Reliability}

Intra-observer reliability was checked by asking the coder to re-code part of the gaze recordings. Two members of each sub-group (e.g., Western novices) were selected for recoding; the first two out of ten minutes re-coded (i.e. 20\%). Intra-class correlation (ICC) of the first coding attempt with the second was deemed acceptable because ICC has been shown to be equivalent to the Fleiss Kappa-the conventional intra-rater reliability measure for nominal data (Fleiss and Cohen 1973; Landis and Koch 1977). The two-way random ICC (ICC-2) was chosen because it was the rater variation that we were concerned with, while the data sampled remained the same (e.g., Bartko 1976). The reliability of our coder was satisfactory (ICC-2 $=0.68$ ).

\section{Scanpath analyses}

Each set of scanpath comparisons resulted in an average similarity score, and our hypotheses required testing whether these similarity scores differed according to the identity, expertise and culture of the source participant. For statistical analyses of similarity scores we ran repeated measures univariate analyses of variance for each didactic behaviour separately (i.e., attentional gaze, then communicative gaze). Transformations were 
conducted prior to running ANOVAs where necessary in order for all dependent variables to meet parametric assumptions, while raw scores are reported for descriptive statistics.

\section{Individual teacher scanpaths}

To address Hypothesis 1, the first set of similarity scores related to comparisons within and between each individual (Table 2). Each participant was thus given a mean similarity score for scanpath comparisons within him or herself as well as a mean similarity score for the scanpath comparisons between him (or her) and others.

Our expectations of top-down guidance for teacher scanpaths in Hypothesis 1 were supported by intra- compared with inter-individual similarities. Within the attentional periods of teaching, intra-individual similarities were significantly greater $(M=0.43)$ than inter-individual similarities $(M=0.38), F(1,39)=29.14, p<0.001, \eta_{p}^{2}=0.43$. Likewise, during communicative gaze, greater scanpath similarities were found in intra-individual $(M=0.40)$ than in inter-individual $(M=0.37)$ comparisons, $F(1,36)=61.34$, $p<0.001, \eta_{p}^{2}=0.63$. Teachers were more similar to themselves in the ordering of their gaze behaviour than they were to each other (Fig. 2). Our subsequent analyses therefore asked whether expertise and culture underlie some of this idiosyncrasy.

\section{Expertise differences in scanpaths}

We expected teacher expertise to be one explanation for differing scanpaths between individuals. The second set of similarity scores related to teacher expertise. We therefore ran string edit comparisons within and across single-IV groupings as shown in Table 3.

Whin-expertise comparisons meant that each teacher was compared to another teacher within their own level of expertise; across-expertise comparisons involve experts being compared with novices. In attentional gaze, teacher scanpath similarity was significantly greater across $(M=0.40)$ than within $(M=0.39)$ expertise, $F(1,39)=15.85, p<0.001$. $\eta_{p}^{2}=0.29$. In communicative gaze, teacher scanpath similarity was greater across $(M=0.39)$ than within $(M=0.38)$ expertise, $F(1,39)=35.16, p<0.001, \eta_{p}^{2}=0.48$. These findings opposed Hypothesis 2 which predicted that scanpaths would be more similar within expertise groupings.

Table 2 Intra- and Inter-Individual comparisons

\begin{tabular}{lll}
\hline IV & Comparison & \\
\cline { 2 - 3 } & Within & Across \\
\hline Individual & Participant $x$ vs. participant $x$ & Participant $x$ vs. Participant $x 1$ \\
& Participant $x$ vs. Participant $y 2$ \\
& Participant $x$ vs. Participant $y 3$ \\
& $\ldots$ \\
& Participant $x$ vs. Participant $y 39$
\end{tabular}

An illustration of how intra- (within) and inter- (across) individual comparison of scanpaths were run. Note that this table only shows a sample of all inter-individual comparisons run: the total number of interindividual comparisons were 39, since the sample overall contained 40 participants 
Fig. 2 The similarity scores when making intra- versus interindividual comparisons in teacher scanpaths. Bars show the mean similarity across all comparisons (with standard error bars) Panel A relates to attentional gaze; Panel B relates to communicative gaze. Same $\mathrm{P}=$ same participant or intra-individual, Diff $\mathrm{P}=$ different participant or interindividual. ${ }^{*} p<0.001$
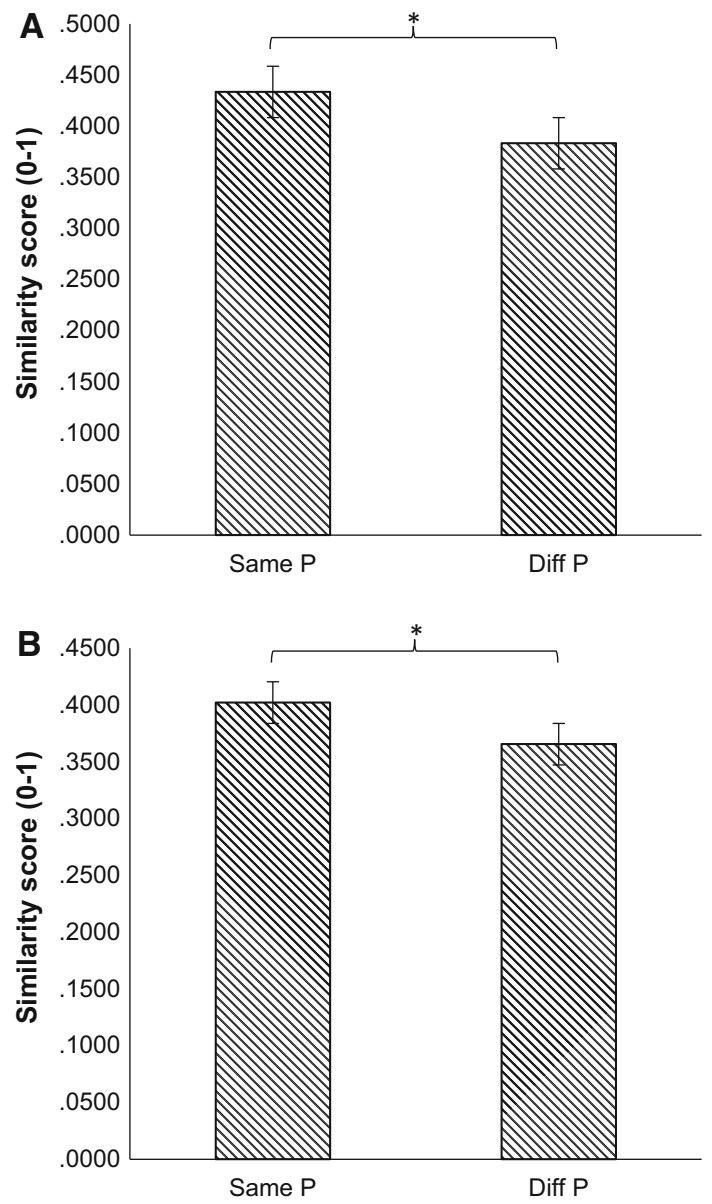

Table 3 Single-IV scanpath comparisons

All the comparisons for deriving single-IV scanpath similarity scores are listed in this table

\begin{tabular}{lll}
\hline IV & Comparison & \\
\cline { 2 - 3 } & Within & Across \\
\hline Expertise & $\begin{array}{l}\text { Expert vs. expert } \\
\text { Novice vs. novice }\end{array}$ & Expert vs. novice \\
Culture & Hong Kong vs. Hong Kong & Hong Kong vs. UK \\
& UK vs. UK & \\
\hline
\end{tabular}

We also compared teacher scanpaths across different expertise but within the same culture through dual-IV groupings, shown in Table 4. Thus, we controlled for culture in the second expertise comparisons.

Once culture was controlled for, within-expertise comparisons meant that each teacher was compared to another teacher within their own level of expertise; across-expertise comparisons meant that experts were compared with novices within the se culture. In attentional gaze, similarity scores were significantly more similar within $(M=0.39)$ than 
Table 4 Dual-IV scanpath comparisons

\begin{tabular}{|c|c|c|c|c|c|}
\hline \multicolumn{3}{|c|}{ Comparison } & \multicolumn{2}{|l|}{ Scanpath 1} & \multirow{2}{*}{$\frac{\text { Scanpath } 2}{\text { Hong Kong Expert }}$} \\
\hline A & Within sub-group & 1 & Hong Kong Expert & $\leftrightarrow$ & \\
\hline & & 2 & UK Expert & $\leftrightarrow$ & UK Expert \\
\hline & & 3 & Hong Kong Novice & $\leftrightarrow$ & Hong Kong Novice \\
\hline & & 4 & UK Novice & $\leftrightarrow$ & UK Novice \\
\hline \multirow[t]{2}{*}{ B } & Within culture, across expertise & 1 & Hong Kong Expert & $\leftrightarrow$ & Hong Kong Novice \\
\hline & & 2 & UK Expert & $\leftrightarrow$ & UK Novice \\
\hline \multirow[t]{2}{*}{$\mathrm{C}$} & Across cultures, within expertise & 1 & Hong Kong Expert & $\leftrightarrow$ & UK Expert \\
\hline & & 2 & Hong Kong Novice & $\leftrightarrow$ & UK Novice \\
\hline \multirow[t]{2}{*}{$\mathrm{D}$} & Across sub-groups & 1 & Hong Kong Expert & $\leftrightarrow$ & UK Novice \\
\hline & & 2 & UK Expert & $\leftrightarrow$ & Hong Kong Novice \\
\hline
\end{tabular}

All the comparisons for deriving dual-IV scanpath similarity scores are listed in this table

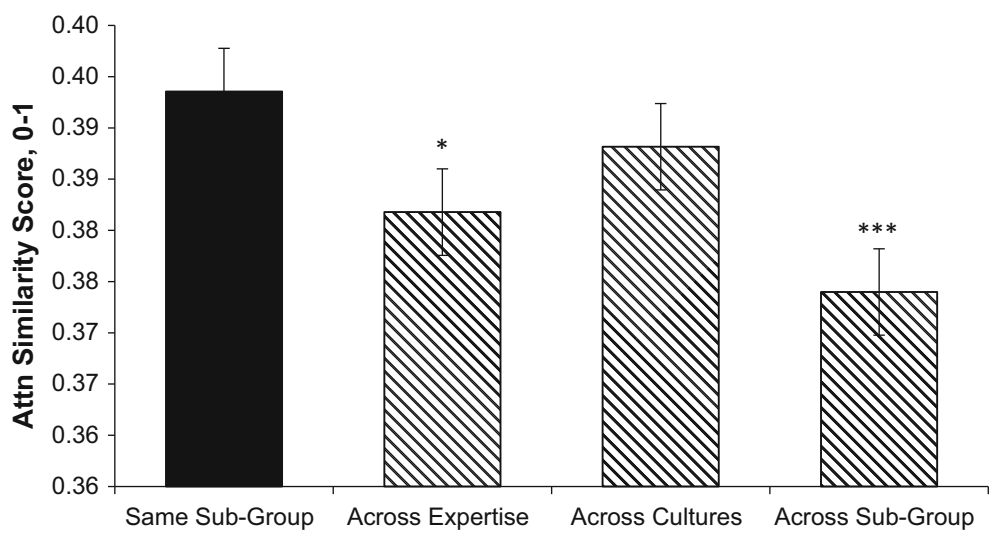

Fig. 3 The dual-IV comparisons conducted in this study on teachers' attentional gaze. Attn = Attentional gaze, Same sub-group = same expertise and cultural group (e.g., Hong Kong experts vs. Hong Kong experts), Across expertise = same culture, different expertise (e.g., Hong Kong experts vs. Hong Kong novices), Across cultures = same expertise, different culture (e.g., Hong Kong experts vs. UK experts), Across sub-group $=$ teacher from differing expertise and culture (e.g., Hong Kong experts vs. UK novices). $* p \leq 0.05, * * p \leq 0.01, * * * p \leq 0.001$ when compared with 'Same sub-group'

across $(M=0.38)$ expertise, $F(1,39)=4.89, p=0.03, \eta_{p}^{2}=0.11$ (Fig. 3). In communicative gaze, scanpaths were more similar within $(M=0.39)$ than across $(M=0.37)$ expertise, $F(1,38)=6.92, p=0.01, \eta_{p}^{2}=0.15$ (Fig. 4). Thus, controlling for culture revealed greater within-expertise similarity than across-expertise similarity in teacher scanpaths, which accorded Hypothesis 2.

\section{Cultural differences in scanpaths}

We expected teacher culture to be another explanation for differing scanpaths between individuals, as stated in Hypothesis 3. We therefore obtained similarity scores for teacher scanpath comparisons within cultures (e.g., UK vs. UK) and across cultures (i.e., Hong 


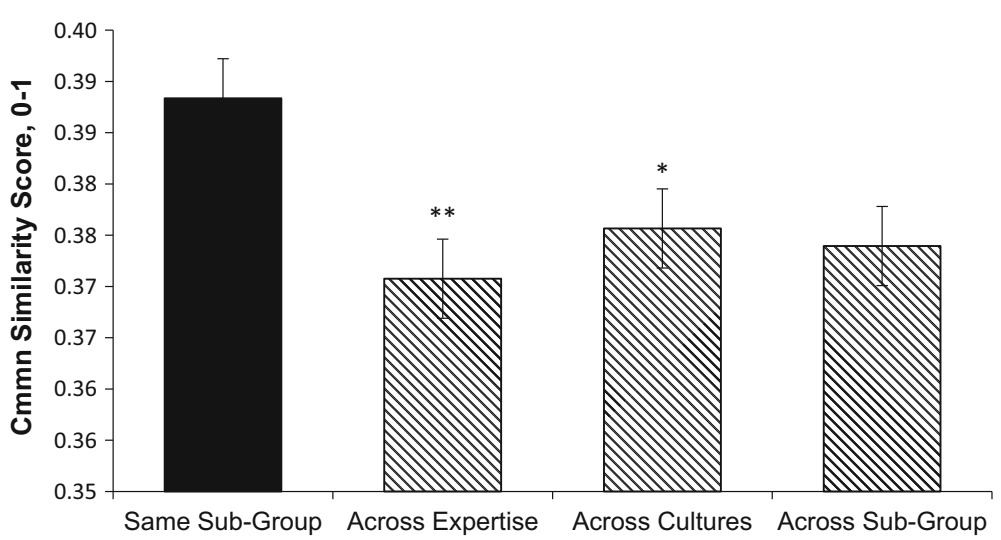

Fig. 4 The dual-IV comparisons conducted in this study on teachers' communicative gaze. Cmmn = communicative gaze, Same sub-group = same expertise and cultural group (e.g., Hong Kong experts vs. Hong Kong experts), Across expertise = same culture, different expertise (e.g., Hong Kong experts vs. Hong Kong novices), Across cultures = same expertise, different culture (e.g., Hong Kong experts vs. UK experts), Across sub-group $=$ teacher from differing expertise and culture (e.g., Hong Kong experts vs. UK novices). $* p \leq 0.05, * * p \leq 0.01, * * * p \leq 0.001$ when compared with 'Same sub-group'

Kong vs. UK; Table 3), without controlling for expertise at this stage (i.e., in single-IV comparisons). In attentional gaze, scanpaths were more similar when compared within $(M=0.39)$ than across $(M=0.38)$ culture, $F(1,39)=5.70 . p=0.02, \eta_{p}^{2}=0.13$. In communicative gaze, scanpaths were more similar within $(M=0.39)$ than across $(M=0.37)$ cultures, $F(1,38)=8.30, p=0.006, \eta_{p}^{2}=0.18$. Both attentional and communicative gaze confirmed Hypothesis 3 at this point.

However, when cultural comparisons of scanpaths were made with expertise controlled for (i.e., in dual-IV comparisons; Table 4), teachers' attentional scanpaths were not more similar within-culture than across $(p=0.08$; Fig. 3), which opposed Hypothesis 3. Nevertheless, teachers' communicative scanpaths accorded Hypothesis 3 by being more similar within $(M=0.39)$ than across $(M=0.38)$ culture, $F(1,38)=3.98, p=0.05, \eta_{\mathrm{p}}^{2}=0.10$ (Fig. 4).

\section{Culture-specific expertise in scanpaths}

We expected teacher expertise and culture to combine and provide the strongest similarity or difference between individuals' scanpaths. To address Hypothesis 4, we compared teacher scanpaths within the same expertise and the same cultural grouping (i.e., same subgroups; Table 4).

Teachers' attentional scanpaths were significantly more similar within $(M=0.39)$ subgroups than across $(M=0.37)$ them, $F(1,39)=11.91, p=0.001, \eta_{p}^{2}=0.23$ (Fig. 3). Communicative scanpaths showed the same effect (within: $M=0.39$; across: $M=0.37$; $F(1,38)=3.83, p=0.06(95 \%$ CI $[-0.16,0.003]), \eta_{p}^{2}=0.09$; Fig. 4), although this did not reach conventional levels of significance. In combination with the other results, both attentional and communicative gaze supported Hypothesis 4 in that scanpaths differed from each other to the greatest extent when originating from individuals from a different culture and a different level of expertise. 


\section{Sub-string descriptions}

After finding that teacher scanpaths differ with expertise and culture (in communicative gaze), we began exploring how teacher scanpaths differ. The most common sequences of teacher scanpaths for each, attentional and communicative, gaze were generated to yield 'sub-strings' of each teacher sub-group (i.e., expertise + culture, e.g., Hong Kong novices). During analysis, a series of trials revealed that a scanpath of six events yielded the greatest difference between the most common (i.e., modal) and second (and/or third) most common scanpaths. These modal scanpaths for each teacher sub-group are shown in Table 5 for each, attention and communication.

Sub-strings revealed relatively minor differences. Experts in both cultures used student fixation first, before alternating between that and less student-oriented gaze, in both attentional and communicative gaze. Since cultural differences were only significant with communicative gaze, we highlight cultural differences therein: namely, Hong Kong teachers used student scan, regardless of expertise, whereas neither experts nor novices used student scan at all in the UK. Culture-specific expertise was shown in both attentional and communicative gaze, with Hong Kong experts using student fixations readily in contrast to Hong Kong novices who used no student fixations. UK experts started with student fixation, whereas novices started with other (i.e., non-student and non-instructional) gaze. Among experts, Hong Kong teachers used student scan whereas UK teachers did not use student scan at all.

\section{Discussion}

The present article sought to demonstrate the role of expertise and culture in the sequencing of teacher gaze. Furthermore, we distinguished between didactic behaviours, analysing attentional (i.e., questioning) gaze separately from communicative (i.e., lecturing) gaze. Our decision to use scanpath comparisons to explore the top-down influences of expertise and culture was supported: participants' gaze sequences were more similar within an individual than when compared between teachers (Hypothesis 1). Dual-IV comparisons controlled for the alternative factor (i.e., grouping system, e.g., culture controlled while making expertise comparisons), which showed attentional scanpaths to be significantly more similar within expertise (Hypothesis 2) and sub-groups (Hypothesis 4), but not culture (Hypothesis 3). Communicative scanpaths, on the other hand, revealed both factors-expertise (Hypothesis 2), culture (Hypothesis 3), and their sub-groupings (Hypothesis 4) - to make a difference to the similarity between gaze sequences. Together, the present study lends strong support for existing frameworks of effective teaching, but does so by showing that the importance of various dimensions of teacher expertise can be seen to the most micro-level of teacher behaviour, namely teacher scanpaths.

\section{Cognitive model for teaching}

Teacher scanpaths were more similar when compared within individuals than across individuals. This finding supports the Scanpath Theory assumption that teachers are guided by top-down rather than bottom-up visual processes, suggesting cognitive models to be active in real-world classroom teaching and driving the order (or sequence) in which 


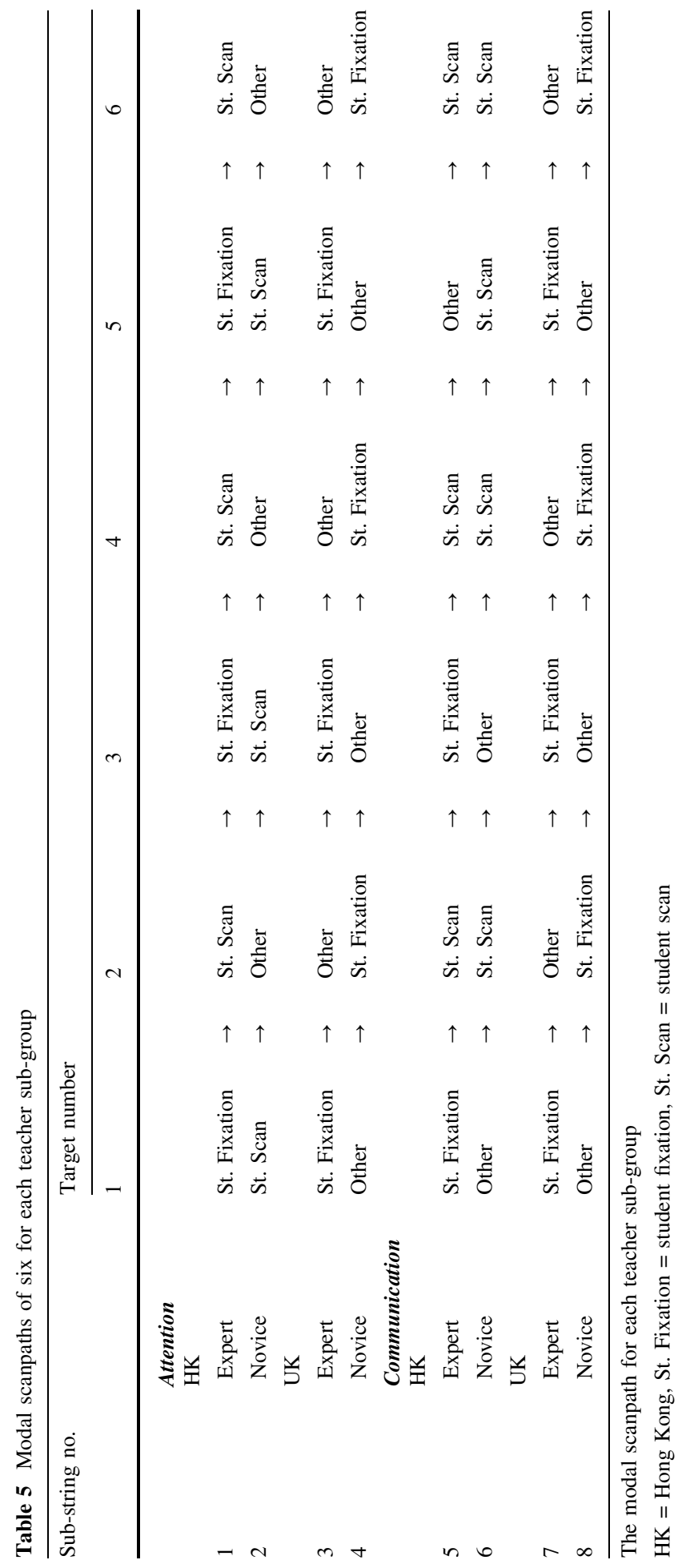


teachers look at different classroom regions. Meanwhile, bottom-up processes are likely to play a less important role in teacher gaze.

It is unsurprising that teachers in this study displayed a top-down process, even at the micro-level of their gaze sequences. Indeed, an overload of top-down processes seems to characterise classroom teaching. Hence Berliner's (2001) comparison of teaching to air traffic control and other high-pressured professions and the rapid exhaustion of cognitive resources among beginning teachers (Berliner 2001). Accordingly, much of their professional development involves identifying the recurrent aspects of teaching for automatic processing, to reserve deliberate cognition for less predictable parts of the profession (Feldon 2007; Van Merriënboer et al. 2002). It is through professional growth that teachers are likely to converge on similar cognitive models for the optimal way of operating in the classroom, even in the minute detail of where they look and the order in which they do so. While we have previously highlighted that experts are more guided by strategy (McIntyre et al. 2017), we presently propose that the content of the cognitive model for classroom teaching is uncovered by identifying expert-novice differences in teachers' scanpaths.

\section{Expertise differences in teacher scanpaths}

The present finding that scanpaths are more similar when compared within than across teacher expertise supports our second hypothesis, that the cognitive model for teacher scanpaths changes with expertise. Specifically, teachers prioritise and order where they look at in the classroom differently when they are experts compared with novice teachers. The sequence of teachers' gaze is therefore a significant indication of professional expertise, quite apart from other measures of the way teachers use their gaze (e.g., summed frequencies or durations). Our finding coincides with research into classroom interactions, which highlights that whole phases of gestural sequences exist as an integral part of the teaching and learning process (e.g., Arnold 2012). Novice teachers can therefore go beyond what they should do more of (e.g., making as much eye contact with students as possible) to give more consideration to the order in which they do things in classroom teaching (e.g., start with eye contact and proceed onto subsequent expert teacher behaviours revealed in their scanpaths).

While the nature of classroom teaching necessitates top-down control, it seems that this process either grows in dominance (i.e., teachers use cognitive models more) or changes in nature (i.e., the content of cognitive models change) as the teacher develops expertise. In terms of the dominance (or importance) of the cognitive model, teacher gaze has shown novice teachers to be more distracted by salient yet task-irrelevant classroom events (e.g., bright shoe laces), whereas expert teacher gaze is guided by pedagogical principles developed over time (e.g., areas surrounding disruptive behaviour, Wolff et al. 2016). Just as gaze behaviour increasingly reflects task-relevant strategy outside the classroom (Haider et al. 2005), so teachers increasingly restrict their gaze to the most task-relevant classroom regions (van den Bogert et al. 2014). The scanpaths of novices in the present study highlight the common errors of beginning teachers. Future novice teachers can learn from the mistakes of the present novice sample by resisting task-irrelevant distractions and focus on the important classroom considerations revealed by expert teachers' scanpaths.

Qualitative analyses suggested that, in both cultures, experts look at students-and return to look at them-more readily than novices do during both attentional and communicative gaze. As such, students constitute a consistent and central component in teachers' gaze sequences. Regardless of culture, experts used student fixation from the beginning and resumed doing so after each diversion. While we had previously 
demonstrated experts' student-centredness through teachers' real-world classroom gaze (McIntyre et al. 2017), we now provide new, additional and direct evidence that expert teachers not only look frequently at students but also show a characteristic sequential pattern of returning to them on subsequent gazes. Our analyses corroborate and extend previous records of student-centredness among expert teachers in contrast to novices' more controlling mind-set both in ours and others' research (Cheon and Reeve 2015; Wolff et al. 2014). Our sequential analyses of teacher gaze thus coincide with existing frameworks of teacher expertise: we highlight that the importance of student-centredness pervades at every level of teacher expertise, beyond existing aggregated analyses of teacher cognition, to the micro-level of analysis such as teacher scanpaths. Novice teachers might give heed to the support from the present study for the centrality of student experience. Rather than focusing on student discipline or salient visual distractions, novices can determine to devote their efforts to the improvement of students' learning experiences and progress.

Our qualitative findings regarding communicative gaze saw experts in both cultures starting with eye contact (i.e., student fixation) then interspersing it with other gaze types (sub-strings 5 and 7 in Table 5). The importance in teachers starting communicative scanpaths by making eye contact with students reflects the natural pedagogical role of eye contact when initiating information transmission (Csibra and Gergely 2009; Frith and Frith 2012) and suggest that teachers are implementing this in the classroom. Indeed, the human eye triggers engagement in the gaze recipient (Committeri et al. 2015; Holler et al. 2014) in a way that artificial stimuli (Ristic et al. 2007) and non-human eyes (Tomasello et al. 2007) cannot. Experts in both cultures appear to be securing student engagement by making eye contact with them first, before shifting their own gaze to another classroom region: an essential gaze sequence for successful shared attention (Baron-Cohen 1995). It seems that the naturally occurring gaze processes of human teaching and learning can indeed be applied to the classroom context. As a result of this study, teachers can apply principles of natural pedagogy and shared attention to their everyday classroom communication and instruction.

\section{The role of culture in expertise differences in teacher scanpaths}

Contrary to preceding analyses (McIntyre et al. 2017), culture on its own failed to predict attentional scanpaths (when expertise was controlled for), culture may be relevant to teacher attentional scanpaths only in relation to attentional expertise. Otherwise, it appears that culture plays less of a role in teachers' attentional scanpaths. Culture is likely to have combined with expertise in correspondence with extant differences between East Asia and Western populations in relationship-orientated vision (Chua et al. 2005) and cognition (Nisbett and Miyamoto 2005).

Both attentional and communicative gaze differed most when teacher scanpaths were compared within sub-groups than when they were compared across sub-groups. Our hypothesis that teachers' expertise and culture would, together, significantly distinguish gaze sequences was supported. This finding corresponds with our previous analysis of the dataset (McIntyre et al. 2017) and existing literature on differing characterisations of effective teaching according to culture (e.g., Zhang et al. 2005). Since culture combines with expertise to affect teachers' cognitive models (i.e., scanpaths), teachers might give greater importance to their specific cultural values when reflecting on best practice for their own classroom context. 
Culture-specific expertise in scanpaths were revealed through qualitative analyses. Qualitative analyses of our teacher sample also demonstrate that scanning gaze occurs during communicative gaze among Hong Kong (or East Asian) teachers exclusively. This finding coincides with previous research highlighting the risk that teacher-student eye contact is offensive (Alston and He 1997), inappropriate (Cheng and Borzi 1997) or intimidating (Akechi et al. 2013) in East Asian settings. Accordingly, it was also expected that teachers would make less use of eye contact to convey that students are welcome to contribute. Even if eye contact is emotionally neutral from teachers, cultures diverge in the meanings signalled by the same gaze patterns (McCarthy et al. 2008). Yet, it was the use rather than the lack of student fixations that set experts apart from novices in Hong Kong, suggesting that teachers opt to exercise authority in East Asia rather than approachability (Hofstede 1986). This finding further echoes existing literature on the universal importance of teacher-student eye contact as part of the communicative episodes during teacher and learning (cf. shared attention, Baron-Cohen 1995, and gaze following, Senju and Csibra 2008). Therefore, while student fixations are a common denominator for experts in both cultures, East Asian culture defines expert scanpaths differently as student fixations are interspersed with student scans among Hong Kong experts only.

\section{Limitations and implications}

In spite of the unique depth of the present investigations into expert teacher gaze, a number of limitations should be acknowledged. Although we contend that scanpaths reflect changing cognitive models, it is important to note that teachers might also differ in their interpretation of what they see. Future research could solicit subjective reports and compare these with gaze records, in order to establish whether teachers exhibiting the same scanpaths are necessarily interpreting the situation in the same way. The present scanpaths were event- rather than duration-based. By taking a duration-based approach to investigating teacher scanpaths, quite a different picture could have emerged, especially regarding teachers' communicative gaze since many of those strings were excluded due to inadequate numbers of events per string. Additionally, most scanpath comparisons yielded noticeably similar scores in spite of significantly greater within- than across-group scanpath similarities. Yet our similarity scores are within the same range as those in extant literature (e.g., Foulsham and Underwood 2008). The scanning gaze coded in the present study should also be interpreted with caution due to the low sampling rate of the eye-tracker $(30 \mathrm{~Hz})$.

We conducted post hoc power analysis to confirm whether our sample size was adequate for the analyses reported in this article. When post hoc power analysis was conducted using G*Power (Erdfelder et al. 1996) for the repeated measures ANOVA prediction of expertise in single-IV comparisons in attentional gaze, the statistical power using our observed effect size $\left(\eta_{p}^{2}=0.29\right)$, sample size $(N=40)$, measurements $(\mathrm{T}=500$; the power does not alter when measurements exceed $\mathrm{T}=500$ ), and the default correlation setting among repeated measures $(r=0.50)$, was determined to be $\beta=0.71$, which neared the standard $\beta=0.80$ power requirement. The repeated measures ANOVA prediction of expertise in dual-IV comparisons of attentional gaze was $\beta=0.11$. Nevertheless, statistically significant findings were found, as reported.

The present article highlights two areas for teacher professional development. First, cognitive models are important in differences between teachers' expertise. The present 
study has given eye-tracking support to the proposal that expert teachers have a cognitive representation distinct from that of novices, which we have revealed through teachers' gaze sequences. That is, teachers change in their operation as they develop professional expertise, both on a macro-level (e.g., Berliner 2001) and on a micro-level-as shown through our participants' gaze sequences. Second, the order in which teachers look at differing regions of the classroom matters, to the extent that expertise manifests not only in summed durations of where teachers look most, but in the sequences of teachers' gaze. Experts in our study demonstrated that their gaze, for both attention and communication, is sequentially distinct from that of novices. Professional development programmes might therefore give closer attention to the cognitive models of expert teachers during questioning (or attention) and lecturing (or communication) via their gaze sequences and give beginning teachers a head start in developing their own, more effective, cognitive models.

Tuse gaze sequences effectively, expert teachers do not develop a universally uniform cognitive model regardless of culture. Rather, the optimal cognitive model in classroom teaching must take culture into consideration. The present study highlights the advantages of culture-specific expertise, as the sequencing of teachers' gaze differs most when both expertise and culture are being compared in scanpath analyses. Thus, the present article corroborates preceding calls for teacher development programs to take cultural context into account: while others have made such calls based on macro-level differences (e.g., student attitudes, Leung 2014; student preferences, Zhang 2006; student emotional experiences, Zhou et al. 2012), our study demonstrates that culture-specificity permeates to the microlevel of effective teacher behaviour in the classroom: namely, their gaze sequences.

Acknowledgements The present research was funded by the Economic and Social Research Council, UK, Grant Number ES/J500215/1. Our gratitude also goes to staff at the Chinese Foundation Secondary School and the Tin Ka Ping Secondary School, Hong Kong, as well as Aston Academy and Eskdale School, UK, for their contribution. In particular, we are indebted to Dr. Lau Kai Shiu, Mr. Tin Wing Sin, Mr. Michael Hall and Mrs. Sue Whelan for all their effort and support.

Open Access This article is distributed under the terms of the Creative Commons Attribution 4.0 International License (http://creativecommons.org/licenses/by/4.0/), which permits unrestricted use, distribution, and reproduction in any medium, provided you give appropriate credit to the original author(s) and the source, provide a link to the Creative Commons license, and indicate if changes were made.

\section{References}

Akechi, H., Senju, A., Uibo, H., Kikuchi, Y., Hasegawa, T., \& Hietanen, J. K. (2013). Attention to eye contact in the West and East: Autonomic responses and evaluative ratings. PLoS ONE, 8(3), e59312.

Allen, R. M., \& Casbergue, R. M. (1997). Evolution of novice through expert teachers' recall: Implications for effective reflection on practice. Teaching and Teacher Education, 13(7), 741-755.

Alston, J., \& He, S. (1997). Business guide to modern China. East Lansing, MI: Michigan State University Press.

Arnold, L. (2012). Dialogic embodied action: Using gesture to organize sequence and participation in instructional interaction. Research on Language \& Social Interaction, 45(3), 269-296.

Bakeman, R., \& Gottman, J. (1997). Observing interaction: An introduction to sequential analysis. Cambridge: Cambridge University Press.

Baron-Cohen, S. (1995). The eye direction detector (EDD) and the shared attention mechanism (SAM): Two cases for evolutionary psychology. In C. Moore \& P. J. Dunham (Eds.), Joint attention: Its origins and role in development (pp. 41-60). New Jersey: Lawrence Erlbaum Associates.

Bartko, J. J. (1976). On various intraclass correlation reliability coefficients. Psychological Bulletin, 83(5), $762-765$. 
Batki, A., Baron-Cohen, S., Wheelwright, S., Connellan, J., \& Ahluwalia, J. (2000). Is there an innate gaze module? Evidence from human neonates. Infant Behavior and Development, 23(2), 223-229.

Bédard, J., \& Chi, M. T. (1992). Current directions in psychological science. Expertise, 1(4), 135-139.

Berliner, D. C. (2001). Learning about and learning from expert teachers. International Journal of Educational Research, 35(5), 463-482.

Berliner, D. C. (2004). Describing the behavior and documenting the accomplishments of expert teachers. Bulletin of Science, Technology \& Society, 24(3), 200-212.

Böckler, A., Eskenazi, T., Sebanz, N., \& Rueschemeyer, S.-A. (2015). (How) observed eye-contact modulates gaze following. An fMRI study. Cognitive Neuroscience, 7, 55-66.

Brandt, S., \& Stark, L. (1997). Spontaneous eye movements during visual imagery reflect the content of the visual scene. Journal of cognitive neuroscience, 9, 27-38.

Chassy, P., \& Gobet, F. (2011). Measuring chess experts' single-use sequence knowledge: An archival study of departure from 'theoretical'openings. PLOS ONE, 6(11), e26692.

Cheng, H., \& Borzi, M. (1997). Chinese students' recognition of typical American cultural situations: Implications for English as a second language instruction. Journal of the Illinois Speech and Theatre Association, 68, 9-22.

Cheon, S. H., \& Reeve, J. (2015). A classroom-based intervention to help teachers decrease students' amotivation. Contemporary Educational Psychology, 40, 99-111.

Choi, Y. S., Mosley, A. D., \& Stark, L. W. (1995). "Starkfest" vision and clinic science special issue: String editing analysis of human visual search. Optometry \& Vision Science, 72(7), 439-451.

Chua, H. F., Boland, J. E., \& Nisbett, R. E. (2005). Cultural variation in eye movements during scene perception. Proceedings of the National academy of Sciences of the United States of America, 102(35), 12629-12633.

Committeri, G., Cirillo, S., Costantini, M., Galati, G., Romani, G. L., \& Aureli, T. (2015). Brain activity modulation during the production of imperative and declarative pointing. Neuroimage, 109, 449-457.

Cortina, K. S., Miller, K. F., McKenzie, R., \& Epstein, A. (2015). Where low and high inference data converge: Validation of CLASS assessment of mathematics instruction using mobile eye tracking with expert and novice teachers. International Journal of Science and Mathematics Education, 13(2), 389-403.

Csibra, G. (2010). Recognizing communicative intentions in infancy. Mind \& Language, 25(2), 141-168.

Csibra, G., \& Gergely, G. (2009). Natural pedagogy. Trends in Cognitive Sciences, 13(4), 148-153.

De Langavant, L. C., Remy, P., Trinkler, I., McIntyre, J., Dupoux, E., Berthoz, A., et al. (2011). Behavioral and neural correlates of communication via pointing. PLoS ONE, 6(3), e17719.

Elliott, J. G., Stemler, S. E., Sternberg, R. J., Grigorenko, E. L., \& Hoffman, N. (2011). The socially skilled teacher and the development of tacit knowledge. British Educational Research Journal, 37(1), 83-103.

Erdfelder, E., Faul, F., \& Buchner, A. (1996). GPOWER: A general power analysis program. Behavior Research Methods, Instruments, \& Computers, 28(1), 1-11.

Ericsson, K. (2014). Expertise. Current Biology, 24(11), R508-R510.

Ericsson, K. A., \& Kintsch, W. (1995). Long-term working memory. Psychological Review, 102(2), 211-245.

Farroni, T., Csibra, G., Simion, F., \& Johnson, M. H. (2002). Eye contact detection in humans from birth. Proceedings of the National Academy of Sciences, 99(14), 9602-9605.

Farroni, T., Massaccesi, S., Pividori, D., \& Johnson, M. H. (2004). Gaze following in newborns. Infancy, $5(1), 39-60$.

Feldon, D. F. (2007). Cognitive load and classroom teaching: The double-edged sword of automaticity. Educational Psychologist, 42(3), 123-137.

Fiske, S. T., \& Taylor, S. E. (2013). Social cognition: From brains to culture. London: Sage.

Fleiss, J. L., \& Cohen, J. (1973). The equivalence of weighted kappa and the intraclass correlation coefficient as measures of reliability. Educational and Psychological Measurement, 33(3), 613-619.

Foerster, R. M., Carbone, E., Koesling, H., \& Schneider, W. X. (2011). Saccadic eye movements in a highspeed bimanual stacking task: Changes of attentional control during learning and automatization. Journal of Vision, 11(7), 1-16.

Foulsham, T., Dewhurst, R., Nyström, M., Jarodzka, H., Johansson, R., Underwood, G., et al. (2012). Comparing scanpaths during scene encoding and recognition: A multi-dimensional approach. Journal of Eye Movement Research, 5(4), 1-14.

Foulsham, T., \& Underwood, G. (2008). What can saliency models predict about eye movements? Spatial and sequential aspects of fixations during encoding and recognition. Journal of Vision, 8(2), 1-17.

Franchak, J. M., Kretch, K. S., Soska, K. C., \& Adolph, K. E. (2011). Head-mounted eye tracking: A new method to describe infant looking. Child Development, 82(6), 1738-1750. 
Freeth, M., Foulsham, T., \& Chapman, P. (2011). The influence of visual saliency on fixation patterns in individuals with Autism Spectrum Disorders. Neuropsychologia, 49(1), 156-160.

Frith, C. D., \& Frith, U. (2012). Mechanisms of social cognition. Annual Reviews of Psychology, 63, 287-313.

Haider, H., Frensch, P. A., \& Joram, D. (2005). Are strategy shifts caused by data-driven processes or by voluntary processes? Consciousness and Cognition, 14(3), 495-519.

Hanley, M., Riby, D. M., Carty, C., Melaugh McAteer, A., Kennedy, A., \& McPhillips, M. (2015). The use of eye-tracking to explore social difficulties in cognitively able students with autism spectrum disorder: A pilot investigation. Autism, 19(7), 868-873.

Henderson, J. M. (2003). Human gaze control during real-world scene perception. Trends in cognitive sciences, 7(11), 498-504.

Hewes, D. E. (1979). The sequential analysis of social interaction. Quarterly Journal of Speech, 65(1), $56-73$.

Hofstede, G. (1986). Cultural differences in teaching and learning. International Journal of Intercultural Relations, 10(3), 301-320.

Holler, J., Schubotz, L., Kelly, S., Hagoort, P., Schuetze, M., \& Özyürek, A. (2014). Social eye gaze modulates processing of speech and co-speech gesture. Cognition, 133(3), 692-697.

Humphrey, K., \& Underwood, G. (2009). Domain knowledge moderates the influence of visual saliency in scene recognition. British Journal of Psychology, 100(2), 377-398.

König, J., Blömeke, S., Paine, L., Schmidt, W. H., \& Hsieh, F.-J. (2011). General pedagogical knowledge of future middle school teachers: On the complex ecology of teacher education in the United States, Germany, and Taiwan. Journal of teacher education, 62(2), 188-201.

Landis, J. R., \& Koch, G. G. (1977). The measurement of observer agreement for categorical data. Biometrics, 33(1), 159-174.

Leinhardt, G. (1987). Development of an expert explanation: An analysis of a sequence of subtraction lessons. Cognition and Instruction, 4(4), 225-282.

Leung, F. K. (1995). The mathematics classroom in Beijing, Hong Kong and London. Educational Studies in Mathematics, 29(4), 297-325.

Leung, F. K. (2014). What can and should we learn from international studies of mathematics achievement? Mathematics Education Research Journal, 26(3), 579-605.

Levenshtein, V. I. (1966). Binary codes capable of correcting deletions, insertions, and reversals. Soviet Physics Doklady, 10(8), 707-710.

McCarthy, A., Lee, K., Itakura, S., \& Muir, D. W. (2008). Gaze display when thinking depends on culture and context. Journal of Cross-Cultural Psychology, 39(6), 716-729.

McIntyre, N. A., Mainhard, M. T., \& Klassen, R. M. (2017). Are you looking to teach? Cultural and dynamic insights into expert teacher gaze. Learning and Instruction, 49, 41-53. https://doi.org/10. 1016/j.learninstruc.2016.12.005.

McNeill, D. (2006). Gesture, gaze, and ground. In S. Renals \& S. Bengio (Eds.), Machine learning for multimodal interaction (pp. 1-14). Edinburgh: Springer.

Nisbett, R. E., \& Miyamoto, Y. (2005). The influence of culture: Holistic versus analytic perception. Trends in Cognitive Sciences, 9(10), 467-473.

Palincsar, A. S. (1998). Social constructivist perspectives on teaching and learning. Annual Review of Psychology, 49, 345-375.

Palmer, D. J., Stough, L. M., Burdenski, J. T. K., \& Gonzales, M. (2005). Identifying teacher expertise: An examination of researchers' decision making. Educational Psychologist, 40(1), 13-25.

Pianta, R. C., Hamre, B. K., \& Allen, J. P. (2012). Teacher-student relationships and engagement: Conceptualizing, measuring, and improving the capacity of classroom interactions. In S. L. Christenson, A. L. Reschly, \& C. Wylie (Eds.), Handbook of research on student engagement (pp. 365-386). London: Springer.

Pianta, R. C., La Paro, K. M., \& Hamre, B. K. (2004). Classrom assessment scoring system. Charlottesville, VA: University of Virginia.

Reingold, E. M., \& Sheridan, H. (2011). Eye movements and visual expertise in chess and medicine. In S. P. Liversedge, I. Gilchrist, \& S. Everling (Eds.), Oxford handbook on eye movements (pp. 767-786). Oxford: Oxford University Press.

Ristic, J., Wright, A., \& Kingstone, A. (2007). Attentional control and reflexive orienting to gaze and arrow cues. Psychonomic Bulletin \& Review, 14(5), 964-969.

Sackett, G. P. (1987). Analysis of sequential social interaction data: Some issues, recent developments, and a causal inference model. New York: Wiley.

Searle, J. R. (1969). Speech acts: An essay in the philosophy of language (Vol. 626). Cambridge: Cambridge University Press. 
Senju, A., \& Csibra, G. (2008). Gaze following in human infants depends on communicative signals. Current Biology, 18(9), 668-671.

Sherin, B. (2006). Common sense clarified: The role of intuitive knowledge in physics problem solving. Journal of research in science teaching, 43(6), 535-555.

Sternberg, R. J. (2014). The development of adaptive competence: Why cultural psychology is necessary and not just nice. Developmental Review, 34(3), 208-224.

Sternberg, R. J., \& Horvath, J. A. (1995). A prototype view of expert teaching. Educational Researcher, 24(6), 9-17.

Tomasello, M. (2000). Culture and cognitive development. Current Directions in Psychological Science, 9(2), 37-40.

Tomasello, M., Hare, B., Lehmann, H., \& Call, J. (2007). Reliance on head versus eyes in the gaze following of great apes and human infants: The cooperative eye hypothesis. Journal of Human Evolution, 52(3), 314-320.

van den Bogert, N., van Bruggen, J., Kostons, D., \& Jochems, W. (2014). First steps into understanding teachers' visual perception of classroom events. Teaching and Teacher Education, 37, 208-216.

van Gog, T., Paas, F., van Merrienboer, J. J., \& Witte, P. (2005). Uncovering the problem-solving process: cued retrospective reporting versus concurrent and retrospective reporting. Journal of Experimental Psychology (Applied), 11(4), 237-244.

Van Merriënboer, J. J., Clark, R. E., \& De Croock, M. B. (2002). Blueprints for complex learning: The 4C/ ID-model. Educational Technology Research and Development, 50(2), 39-61.

Wolff, C. E., Jarodzka, H., Van den Bogert, N., \& Boshuizen, H. P. A. (2016). Teacher vision: Expert and novice teachers' perception of problematic classroom management scenes. Instructional Science, 44(3), 243-265.

Wolff, C., van den Bogert, N., Jarodzka, H., \& Boshuizen, H. P. A. (2014). Keeping an eye on learning: Differences between expert and novice teachers' representations of classroom management events. Journal of Teacher Education, 66(1), 68-85.

Wozniaková, Z. (2016). Different approaches to the Czech and Chinese university students in Business Economics: A teaching experience. Perspectives in Science, 7, 52-58.

Wu, D. W.-L., Bischof, W. F., \& Kingstone, A. (2014). Natural gaze signalling in a social context. Evolution and Human Behavior, 35, 211-218.

Wubbels, T., Brekelmans, M., Mainhard, T., den Brok, P., \& van Tartwijk, J. (2016). Teacher-student relationships and student achievement. In K. R. Wentzel \& G. B. Ramani (Eds.), Handbook of social influences in school contexts: Social-emotional, motivation, and cognitive outcomes (pp. 127-145). New York: Routledge.

Yang, M., \& Cobb, P. (1995). A cross-cultural investigation into the development of place-value concepts of children in Taiwan and the United States. Educational Studies in Mathematics, 28(1), 1-33.

Zhang, L.-F. (2006). Preferred teaching styles and modes of thinking among university students in mainland China. Thinking Skills and Creativity, 1(2), 95-107.

Zhang, L.-F., Huang, J., \& Zhang, L. (2005). Preferences in teaching styles among Hong Kong and US university students. Personality and Individual Differences, 39(7), 1319-1331.

Zhou, N., Lam, S.-F., \& Chan, K. C. (2012). The Chinese classroom paradox: A cross-cultural comparison of teacher controlling behaviors. Journal of Educational Psychology, 104(4), 1162-1174. 